\title{
A time-series analysis of changeover performance on concurrent variable-interval schedules
}

\author{
PAMELA G. REAL \\ Adelphi University, Garden City, New York
}

\begin{abstract}
Pigeons responded to concurrent variable-interval variable-interval schedules of food reinforcement. Interchangeover times and reinforcements were recorded in intact time series during acquisition following several schedule-density manipulations. Probability of a changeover, while constant as a function of time since the previous changeover, was found to be a nonmonotonically increasing function of time since reinforcement. Autocorrelation analyses of intact time series revealed the presence of statistically significant sequential dependencies in the time series during acquisition, but not during asymptotic performance. Lag transformations of actual interchangeover times revealed inhomogeneities in the time series not shown in the autocorrelations or in the overall distribution of interchangeover times.
\end{abstract}

The character of selections (changeovers, COs) among alternatives of a concurrent schedule has been the focus of recent attention (Heyman, 1979a, 1979b; Heyman \& Luce, 1979; Shimp, 1969, 1975; Silberberg, Hamilton, Ziriax, \& Casey, 1978). There has, to date, been little consensus achieved with regard to the type of reinforcement processes that maintain $\mathrm{CO}$ performance. Theoretical disagreements aside, the few accounts relating molecular-level $\mathrm{CO}$ performance to some other aspect of concurrent schedule behavior have demonstrated some empirical consistencies. Hinson and Staddon $(1981,1983)$, Menlove (1975), and Silberberg et al. (1978) have found that there is often a modal amount of time between successive COs (interchangeover time, ICT). Highly unimodal distribution of ICTs appears to be particularly characteristic of performance on the least preferred of an asymmetrical concurrent variableinterval variable-interval pair. Furthermore, these studies have found that local time allocation is much more variable in the preferred of an asymmetrical pair. Silberberg et al. have found that probability of a $\mathrm{CO}$ as a function of time, at least in the preferred component, is roughly constant.

Reports of $\mathrm{CO}$ performance as a function of run length are slightly less consistent. Silberberg et al.

This paper is based, in part, on a dissertation submitted to the Graduate School of Arts and Sciences, Adelphi University, in partial fulfillment of the requirements for the $\mathrm{PhD}$ degree. Portions of this experiment were presented at the annual meeting of the Eastern Psychological Association, Philadelphia, 1979. The author is now at the Department of Psychology, Middlesex House, University of Massachusetts, Amherst, Massachusetts 01003. Thanks are extended to Ted Cochran and Deb Olson for their helpful comments. found slight increasing probabilities of a $\mathrm{CO}$ as a function of run length, in most cases, when a changeover delay (COD) was employed. However, without a COD, the conditional probability of a $\mathrm{CO}$ to the preferred of an asymmetrical pair, initially high, decreased monotonically after runs of more than two responses to the unpreferred component. Similar data for COs to the unpreferred component were not reported, but probability of a response to the preferred component was roughly constant after all run lengths on that schedule. Heyman (1979a) and Hinson and Staddon (1983) found that probability of a $\mathrm{CO}$ as a function of run length conformed to the general predictions of a Markov model. That is, CO probabilities were constant and independent of the number of responses since the previous $\mathrm{CO}$.

Concerning the relationship between reinforcement and $\mathrm{CO}$ responding, Menlove (1975) reported that the majority of reinforcements on concurrent variable-interval variable-interval schedules occurred immediately after the COD. Dreyfus, Dorman, Fetterman, and Stubbs (1982) have demonstrated a systematic relationship between the percent of reinforcements arranged for an unattended component, and therefore scheduled for delivery after the first response to that component that meets any existing delay contingency, and relative amount of time allocated to available components. Hinson and Staddon (1983) have shown that, in spite of this potentially discriminable local pattern of reinforcement, there is little difference in the probability of a response to either schedule following reinforcement.

The emergence of some consistency is encouraging and suggests that empirical agreement, on some level, across studies and procedural variations is not a complete exception to the rule, as some theorists have 
suggested (Herrnstein \& Loveland, 1975; Nevin, 1979). The description of molecular-level CO performance is, however, not yet complete. Overall conditional probability as a measure of $\mathrm{CO}$ performance implicitly limits the determinants of choice to some aspect of performance since the immediately preceding $\mathrm{CO}$. Therefore, systematic relationships between $\mathrm{COs}$ in the time series remote from one another may not be apparent. Furthermore, the occurrence of reinforcement is infrequent relative to total behavioral output on a typical concurrent schedule. Potential local effects of reinforcement on $\mathrm{CO}$ performance would be averaged in assessments of overall CO probability that favor ICTs during which reinforcement fails to occur, by virtue of their greater frequency. Finally, it is not clear from analyses of asymptotic performance on concurrent schedules whether or not there are systematic molecular-level changes in performance during acquisition. The present study reports the results of analyses of intact time series of ICTs and reinforcements during acquisition following several schedule-density manipulations.

\section{METHOD}

\section{Subjects}

Three male Carneaux pigeons, maintained at $80 \%$ of their freefeeding weights, served as subjects. All subjects had had previous exposure to concurrent reinforcement schedules.

\section{Apparatus}

A standard three-key pigeon pecking-panel (BRS-LVE) was mounted in a sound-attenuated chamber, which provided an experimental enclosure $36 \mathrm{~cm}$ high, $35 \mathrm{~cm}$ wide, and $30 \mathrm{~cm}$ deep. Only the center and right keys were used. The keys were mounted $8.3 \mathrm{~cm}$ apart and $25 \mathrm{~cm}$ from the floor of the chamber. The keys were transilluminated by 28-Vdc three-color stimulus units (BRSLVE). A force of approximately $0.1 \mathrm{~N}$ was required to operate the keys. The opening of the grain magazine was directly below the center key and $10.3 \mathrm{~cm}$ from the floor. Masking noise was provided through speakers in the chamber and in the room housing the chamber. Standard 28-Vdc electromechanical programming equipment arranged the experimental events and recording from an adjacent room.

\section{Procedure}

During the first phase of the experiment, the subjects were reinforced on a CO-key concurrent variable-interval 120 -sec variableinterval 120-sec schedule (intervals for the VI tape were prescribed by Fleshler \& Hoffman, 1962). Each response to the white center key (CO key) alternated the schedules, the right-key (main-key) stimuli (red and green), and initiated a 2 -sec COD. Main-key responses were reinforced according to the prevailing schedule by 3 sec access to mixed grain. Sessions were terminated following 60 reinforcements.

The subjects were then exposed to the following order of schedule manipulations: concurrent VI $120 \mathrm{sec}$ (red) VI $60 \mathrm{sec}$ (green); concurrent VI $80 \mathrm{sec}$ VI $80 \mathrm{sec}$; concurrent $60 \mathrm{sec}$ (red) VI $120 \mathrm{sec}$ (green). Table 1 shows the number of sessions in each condition for all three subjects.

\section{RESULTS}

Figures 1 and 2 show the relative frequency of ICTs that terminated in 30 consecutive 1 -sec class intervals. The figures show acquisition following all schedule manipulations in their order of presentation for Bird 416. In general, as the reinforcement schedule associated with the red component varied from VI $120 \mathrm{sec}$ to VI $80 \mathrm{sec}$ to VI $60 \mathrm{sec}$, ICTs in the red component increased in duration and variability, whereas ICTs in the green component became briefer and less variable as the reinforcement schedule associated with the green component varied from VI $60 \mathrm{sec}$ to VI $80 \mathrm{sec}$ to VI $120 \mathrm{sec}$. Early sessions of acquisition often showed decreases in the variability and duration of ICTs. Occasionally, there were changes in a direction opposite to what would have been predicted on the basis of the schedule change [note early sessions of concurrent VI $80 \mathrm{sec}$ VI $80 \mathrm{sec}$

Table 1

Number of Sessions per Condition, Responses, Time, and Reinforcements in Red and Green Components and Total COs

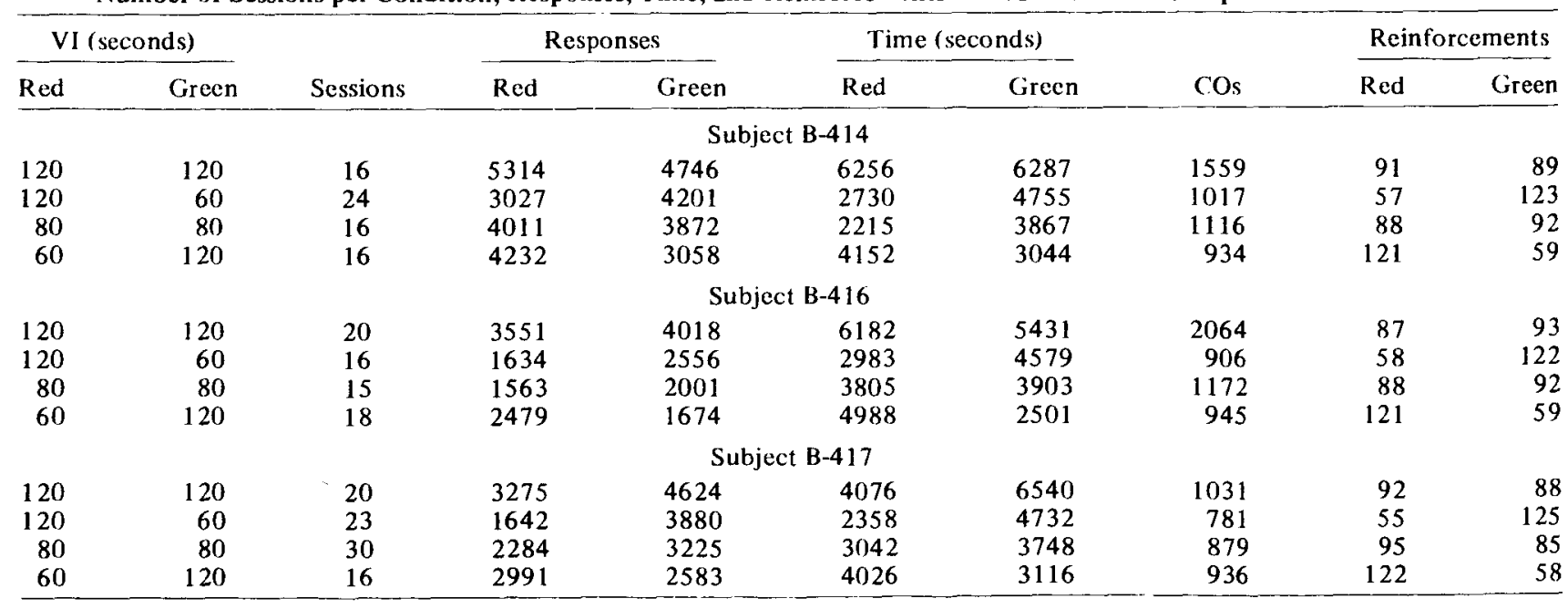

Note-Data are summed over the last three sessions per condition. 


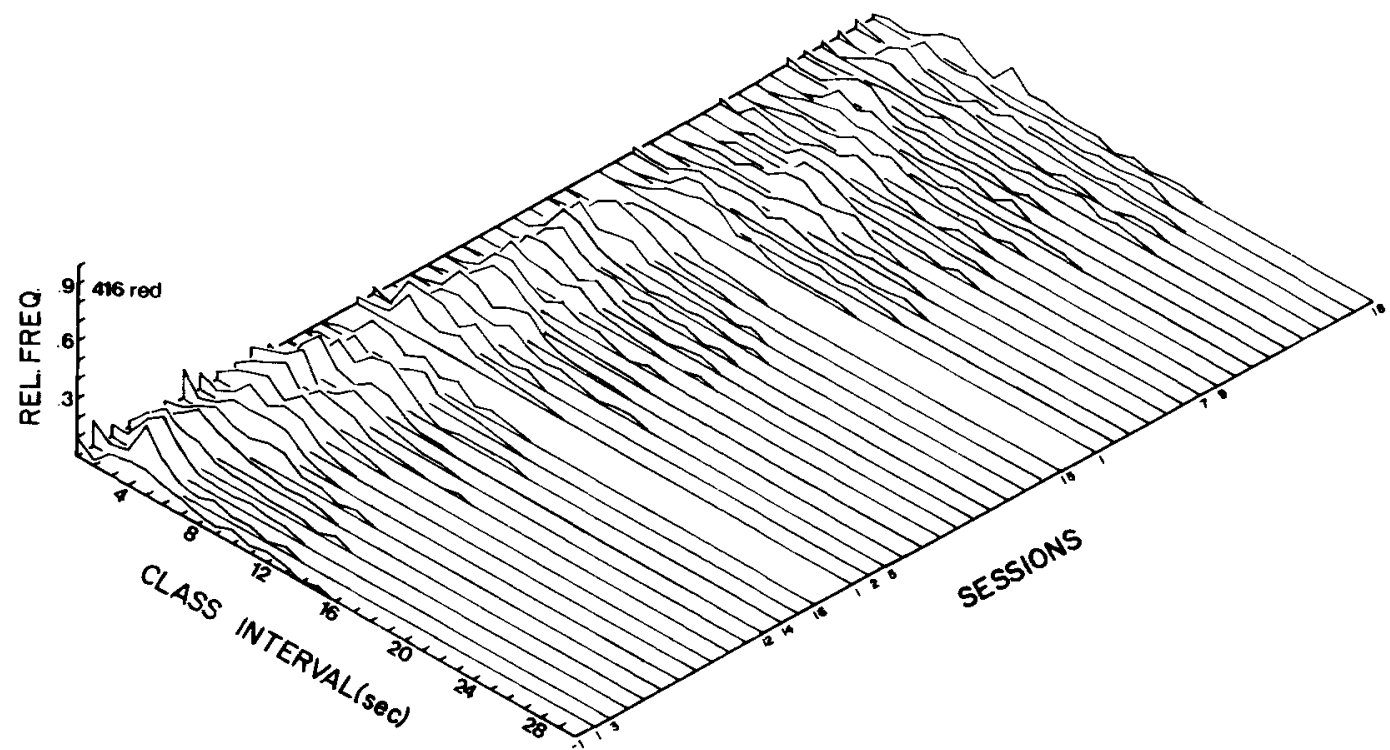

Figure 1. Relative frequency of ICTs in the red component during acquiaition after all schedule changes for Bird 416. Seasions are plotted on the $X$ axd, relative frequency on the $Y$ axdis, and the upper limit of 30 consecutive 1-sec clan intervals on the $Z$ axds. The last sesulon of concarrent VI 120 sec VI 120 sec bs habeled -1 , and all other seations are labeled unless condguous. Seadons from each condition are separated from one another and presented in order (see Table 1).

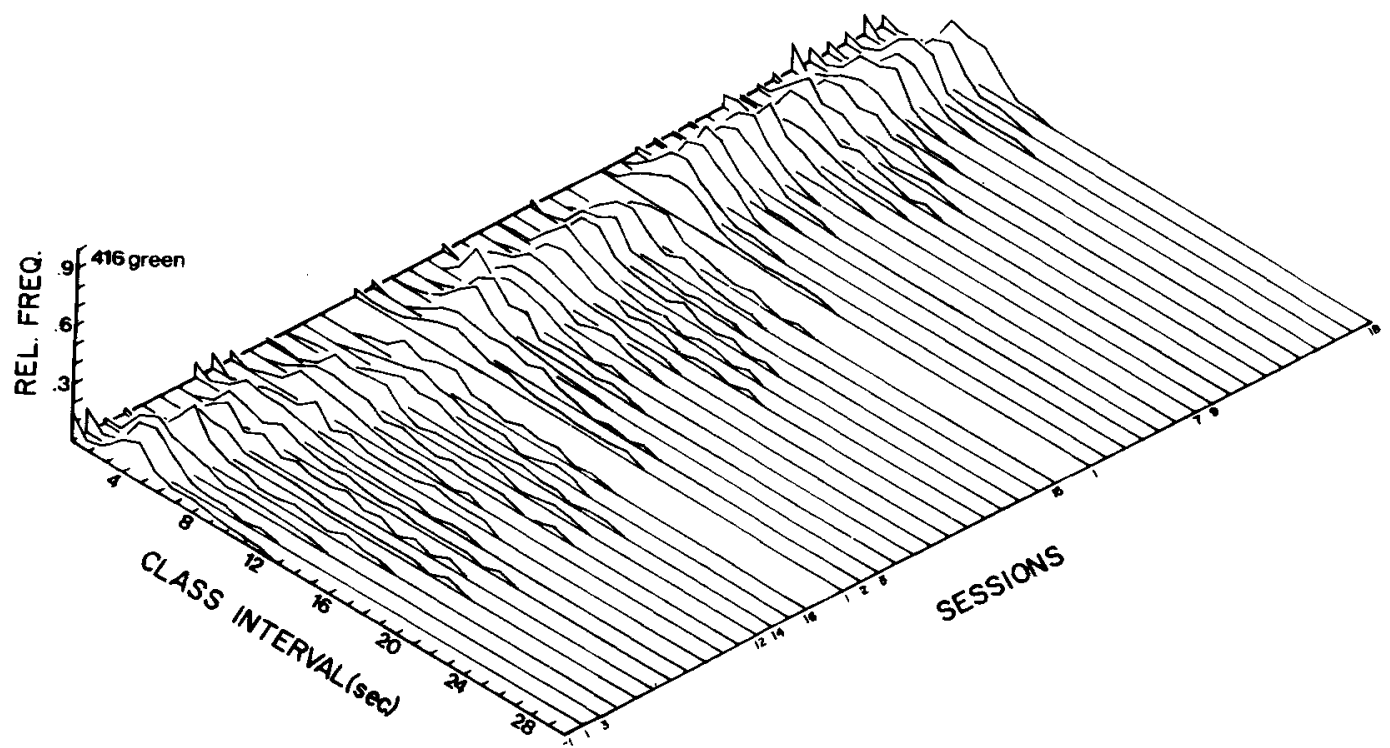

Ftgare 2. Relative frequency of ICTs in the green component durhg acquibition after all schedule changes for Bird 416 (see detalls in Figure 1 caption).

in the red component; early sessions of concurrent VI $120 \mathrm{sec}$ (red) VI $60 \mathrm{sec}$ (green) in the green component]. When the reduction in duration and variability was in the same direction as would have been predicted by the schedule change, it was often in excess of what would be predicted on the basis of terminal performance in a condition [note early sessions of concurrent VI $120 \mathrm{sec}$ (red) VI $60 \mathrm{sec}$ (green) and concurrent VI $80 \mathrm{sec}$ VI $80 \mathrm{sec}$ in the red component; early sessions of concurrent VI $60 \mathrm{sec}$ (red) VI $120 \mathrm{sec}$ (green) in the green component]. These reductions in variability were characteristic of all subjects.

Figure 3 shows lag relationships between ICTs in the green component for the same subject. The last session of concurrent VI $120 \mathrm{sec}$ VI $120 \mathrm{sec}$ and the 

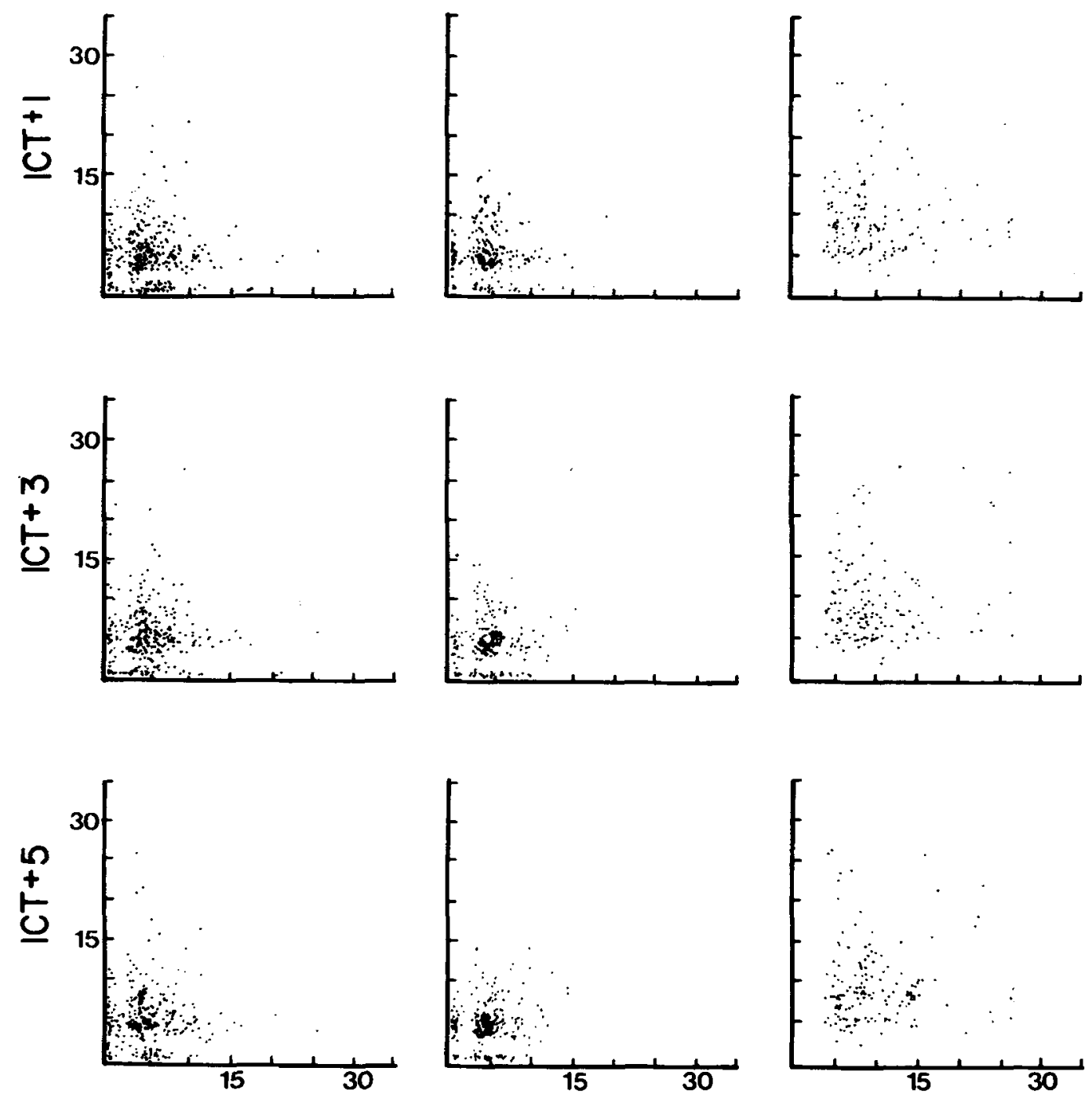

\section{ICT(sec)}

Figure 3. The duration of an ICT $+n$ (in seconda) plotted as a function of ICT (in seconds) for Bird 416. The last seasion of concurrent VI $120 \mathrm{sec}$ VI $120 \mathrm{sec}$ and the first and last sessions of concurrent VI $120 \mathrm{sec}$ (red) VI $60 \mathrm{sec}$ (green) are displayed in columns.

first and last sessions of concurrent VI $120 \mathrm{sec}$ (red) VI $60 \mathrm{sec}$ (green) are displayed in columns. ICT is plotted on the abscissa, and the duration of ICT $+n$ (where $n$ is the integer 1,3 , or 5 ) is plotted on the ordinate. So, for example, in the first row, the durations of ICT pairs 1 and 2,2 and 3,3 and $4, \ldots$, etc., in the time series form a set of $(x, y)$ coordinates. The first two columns show features common to many of the sessions that were analyzed. The first feature is the cluster of data points close to the axes, indicating very brief ICTs. These brief ICTs tended to be characteristic of performance in the less preferred of an asymmetrical pair of schedules and of performance during early sessions of acquisition for all subjects. The second feature is the absence of data points in two distinct bands that parallel the axes, representing the approximate duration of the CODs, during which few COs were made. The tendency for data points to cluster parallel to the axes (excluding very brief ICTs) reflects the presence of a modal ICT. These most frequently occurring durations tended to dominate all analyses of sessions showing clearly unimodal relative frequency distributions. However, in the last session of concurrent VI $120 \mathrm{sec}$ VI $120 \mathrm{sec}$ and the first session of concurrent VI $120 \mathrm{sec}$ (red) VI $60 \mathrm{sec}$ (green), the tendency to parallel the axes is more pronounced at lags 1 and 3 than at lag 5. At lag 5, more data points cluster 
along a hypothetical 45-deg diagonal drawn from the origin. This suggests that ICTs of more similar duration tended to follow one another at lag 5 and that the characteristics of the time series at lag 1 were not preserved at larger lags. During the last session of concurrent VI $120 \mathrm{sec}$ (red) VI $60 \mathrm{sec}$ (green), the increased variability of ICTs is clear, as is the absence of very brief ICTs. The tendency for data points to parallel the axes at lag 1 and to approach the diagonal at lags 3 and 5 is present, but less pronounced.

Figure 4 shows the relative frequency with which reinforcement occurred in the red component as a function of time since a $\mathrm{CO}$ response. Time since a $\mathrm{CO}$ is divided into 15 consecutive 1 -sec class intervals. The data are from selected sessions of acquisition for all phases of the experiment for all three subjects. The most remarkable feature is that the temporal distribution of reinforcement was virtually invariant over the course of the experiment. The majority of reinforcements were delivered within the first second after the COD during all phases. Sessions in which the distribution of ICTs appeared transitional (see Figures 1 and 2) are not differentiable on the basis of temporal distribution of reinforcement. This was true for all subjects and in both components.

Figure 5 shows probability of a $\mathrm{CO}(1)$ as a function of ICT for all ICTs less those in which reinforcement occurred and (2) as a function of time since reinforcement for those ICTs during which reinforcement occurred in both main-key com- ponents. Data displayed are averages of conditional probabilities in the first 12 consecutive 1 -sec class intervals from the last three sessions per condition. Conditional probability was determined by dividing the frequency of ICTs terminating in an interval by the frequency of ICTs terminating in that and subsequent intervals. Data from the red component are shown by the squares, data from the green component are shown by the circles.

Probability of a $\mathrm{CO}$ as a function of ICT was higher in the less preferred of an asymmetrical pair of schedules, increased gradually in the first 2-5 class intervals, and thereafter appeared roughly constant. During concurrent VI $80 \mathrm{sec}$ VI $80 \mathrm{sec}$, one subject (417) showed increasing probability of a $\mathrm{CO}$ as a function of time in the green component. Probability of a $\mathrm{CO}$ as a function of time since reinforcement increased nonmonotonically in 13 of 18 cases. When conditional probability of a $\mathrm{CO}$ following reinforcement appeared roughly constant in the asymmetrical pairs, it was always in the preferred component [i.e., Birds 416 and 417 in the green component during concurrent VI $120 \mathrm{sec}$ (red) VI $60 \mathrm{sec}$ (green) and Bird 417 in the red component during concurrent VI $60 \mathrm{sec}$ (red) VI $120 \mathrm{sec}$ (green)]. Conditional probability of a $\mathrm{CO}$ as a function of time since reinforcement appeared unsystematically related to schedule density in the case of two subjects (416, 417) during concurrent VI $80 \mathrm{sec}$ VI $80 \mathrm{sec}$. Given the obvious pattern in Figure 4, time to reinforcement following a $\mathrm{CO}$ was roughly constant. Dif-

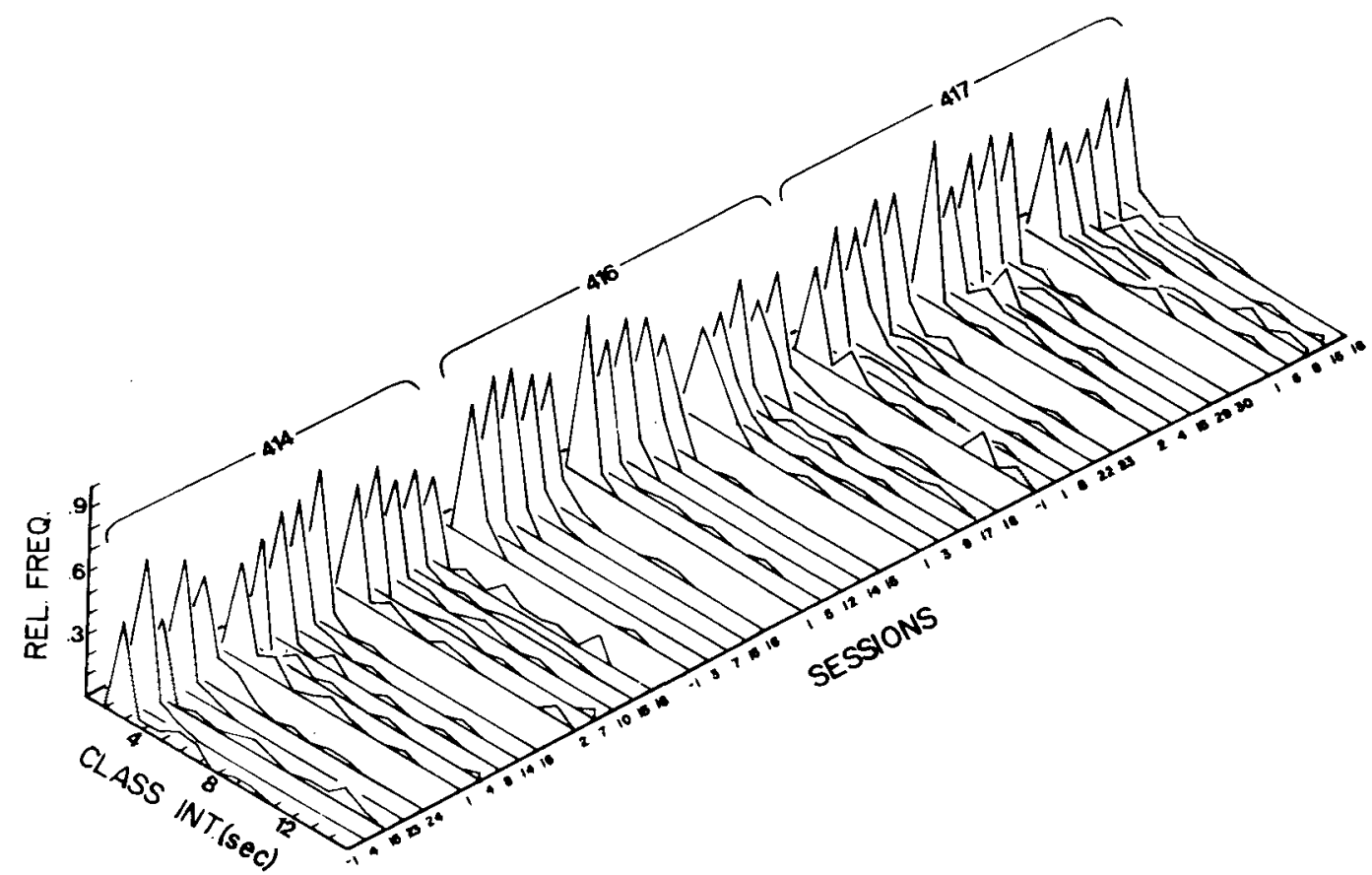

Figure 4. Relative frequency with which reinforcement occurred in the red component as a function of time since $a \mathrm{CO}$ to the red component for selected sessions of acquisition for all subjects. Seasions are plotied on the $X$ axis, relative frequency on the $Y$ axis, and upper limit of 15 consecutive 1-sec class intervals on the $Z$ axis. Sessions from each condition are separated from one another and presented in order (Table 1). 

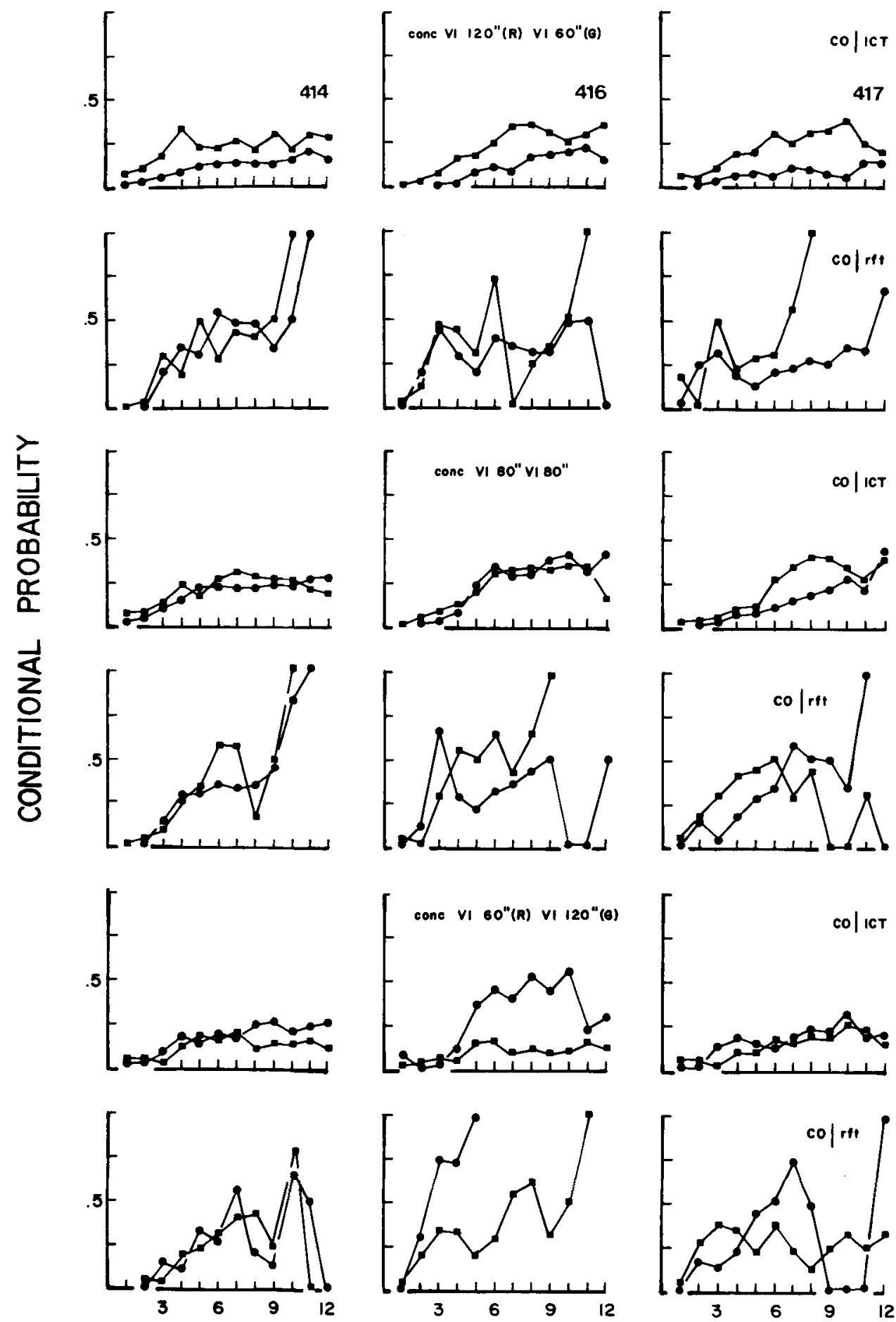

CLASS INTERVAL(sec)

Figure 5. Conditional probability of a CO during Phases $2-4$ for all subjects. Upper limit of 12 consecutive 1-sec class intervals is plotted along the $Y$ axis. Columns represent the individual subjects. Row 1 of each pair shows conditional probability of a CO as a function of ICT duration less those ICTs in which reinforcement occurred. Row 2 of each pair shows conditional probability of a CO as a function of time since reinforcement in an ICT. Squares show the dats from the red component, and circles show the data from the green component. 

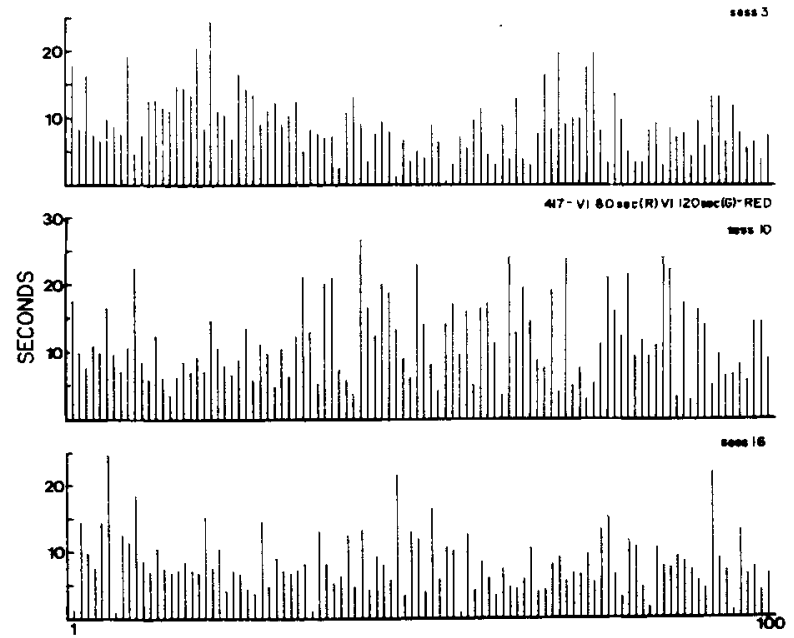

ORDINAL POSITION

Figure 6. The first 100 ICT: in the red component from selected sesolons of concurrent VI $60 \mathrm{sec}$ (red) VI $120 \mathrm{sec}$ (green) for Bird 417.
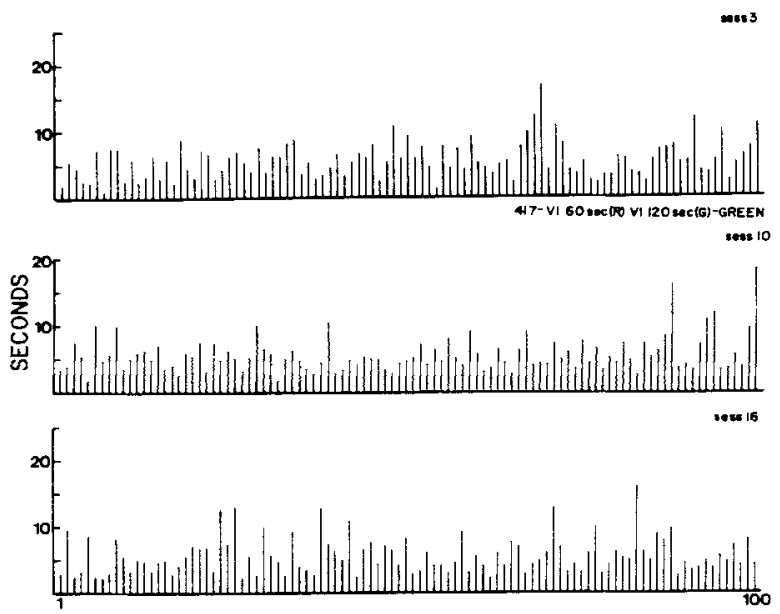

ORDINAL POSITION

Figure 7. The first 100 ICTs in the green component from selected sessions of concurrent VI $60 \mathrm{sec}$ (red) VI $120 \mathrm{sec}$ (green) for Bird 417.

ferences between overall $\mathrm{CO}$ probabilities and $\mathrm{CO}$ probabilities as a function of time since reinforcement are, therefore, attributable to the characteristics of staying times after reinforcement.

More detailed inspection of the data revealed that there were features of the time series that failed to emerge in the summary statistics. Figures 6 and 7 show the first 100 ICTs from the actual intact time series from both main-key components for Sessions 3,10 , and 16 of concurrent VI $60 \mathrm{sec}$ (red) VI $120 \mathrm{sec}$ (green) for one subject (417). Interchangeover times in the red component were clearly more variable and of overall greater duration than those in the green component, similar to the trends in Figures 1 and 2. ICTs also appeared in cyclic patterns, or in ascend- ing and descending sequences of approximately 5-6 ICTs. The first 30 ICTs in the red component from Session 10 show this type of pattern clearly; a similar, but less pronounced, trend is apparent in this session in the green component. Interchangeover times at the beginning of Session 16 (red component) appear to be more variable and of greater duration than later ICTs.

Autocorrelation provides a method of assessing the relationship between successive observations within a time series by computing the covariation of $X_{i+L}$ and $X_{i}$, where $X$ is the value of observation $i$ and $L$ is some number of observations, or lags, removed. Hence, at $L=1$, a covariation is computed between series $X_{1}, X_{2}, X_{3}, \ldots, X_{n-1}$ and series $X_{2}, X_{3}$, $X_{1}, \ldots, X_{n}$, where all $X s$ are part of the same series and ordered in time or space (Weiss, Laties, Siegal, \& Goldstein, 1966). The normalized autocorrelation coefficient at lag $\mathrm{L}$ for a series of $\mathrm{N}$ observations is given by:

$$
\begin{aligned}
{ }_{L} R_{N} & =\frac{{ }_{L} C_{N}}{V_{N}} \\
& =\frac{X_{1} X_{L+1}+X_{2} X_{L+2}+\cdots X_{N} X_{L}-\left(\Sigma X_{i}\right)^{2} / N}{\Sigma X_{i}^{2}-\left(\Sigma X_{i}\right)^{2} / N},
\end{aligned}
$$

where $\mathrm{C}$ is covariance and $\mathrm{V}$ is variance (Anderson, 1942). Hence, at $L=0,{ }_{0} R_{N}=1$. Constraints on the use of this statistic prohibit assessment of autocorrelation at lags exceeding $10 \%$ of the total number of observations within the time series (Blackman \& Tukey, 1958). Obtained autocorrelation coefficients can be compared with the predictions of a Markov process, in which the change in behavior at any time is constant and independent of its history. A Markov process would yield autocorrelation coefficients at any $L$ by ${ }_{L} R_{N}={ }_{1} R_{N}^{L}$, or an exponential decay relative to ${ }_{1} R_{N}$ (Weiss et al., 1966).

Tests of statistical significance have been derived for ${ }_{1} R_{N}$ (Anderson, 1942; Tintner, 1952) on the assumption that it is normally distributed with a mean of $-1 /(N-1)$ and variance $(N-2) /(N-1)^{2}$. For large samples, significance levels for ${ }_{1} R_{N}$ yield good approximations of significance levels at any $L$ (Anderson, 1942). However, computation of statistical significance depends upon the number of items in each series. Since the number of items in each of the time series tested in this experiment differed, the single-tail significance levels for $\mathbf{N}=75$ were adopted as a conservative estimate, since $\mathbf{N}$ exceeded 75 in all cases. At $\mathrm{N}=75$, the $5 \%$ and $1 \%$ levels of significance for the positive tail are 0.173 and 0.250 , respectively. For the negative tail, $5 \%$ and $1 \%$ levels of significance are -0.199 and -0.276 , respectively.

Intact time series for all subjects from each phase of the experiment were subjected to autocorrelation 
analysis up to $\mathrm{L}=10$. ICTs from each main-key component were treated as independent series and analyzed separately. The data were corrected for the presence of linear trends (cf. Weiss et al., 1966) prior to analysis. Figures 8 and 9 show autocorrelograms for selected sessions of concurrent VI $60 \mathrm{sec}$ (red) VI $120 \mathrm{sec}$ (green). The final session of this phase is shown for all subjects. $R=0$ is shown by the horizontal solid line, estimates of positive- and negativetail 5\% significance levels are shown by the horizontal dashed lines.

Performance during concurrent VI $60 \mathrm{sec}$ (red) VI $120 \mathrm{sec}$ (green) was typical of performance during other experimental phases. All subjects showed statistically significant positive and negative correlations during some sessions of each phase. Significant correlations, when they occurred, were typically at $L=1$ or at late lags, that is, $L=6-10$. Significant autocorrelations appeared frequently during acquisition, but not during terminal performance in each phase. With few exceptions, the autocorrelation coefficients generated from analyses of terminal performance approximated, by visual inspection, the predictions of a Markov model.

Bird 417 showed the highest autocorrelation coefficients. In some sessions, autocorrelations were as high as 0.40 . For example, in the green component (Figure 9), Sessions 6 and 10 showed high autocorrelations at $L=6$. This represents a dramatic departure from what would be predicted on the basis of a Markov description of CO performance. However, although Session 10 showed visually obvious periodicity in the time series from the red component, statistically significant autocorrelations failed to emerge at longer lags. One subject (416) showed statistically significant autocorrelations early in acquisition, that is, in Sessions 1-5. The significant autocorrelation at $\mathrm{L}=9$ in the red component during Session 1 , for example, is unlikely to have been the result of the intrusion of high autocorrelations at earlier lags, since coefficients at all preceding lags were approximately zero.

In an effort to determine what the high autocorrelation coefficients in the green component (417) may have been due to, scatter plots of Session 6 were analyzed. Figure 10 shows actual lag relationships $(1,5$, and 6$)$ from this session. The lag 5 relationship, which showed a significant autocorrelation coefficient, shows a band near the 45-deg diagonal in which few data points fell, suggesting that ICTs of similar durations rarely followed one another at lag 5 . The lag 6 relationship, however, which also showed

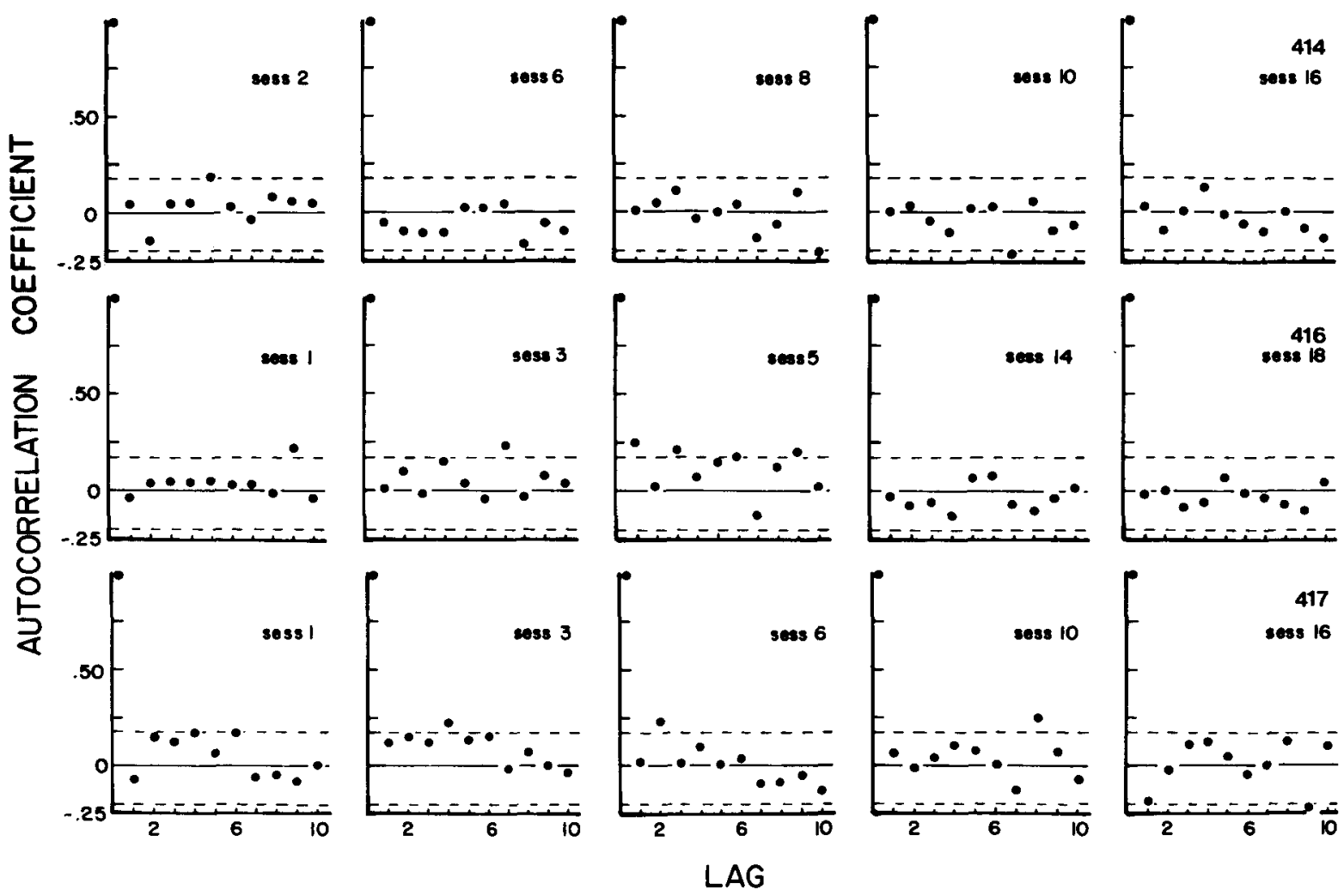

Figure 8. Autocorrelations up to a lag of 10 for selected time series in the red component during acquisition of concurrent VI $60 \mathrm{sec}$ (red) VI $120 \mathrm{sec}$ (green) for all subjects. Rows represent individual subjects. The horizontal solid line shows $R=0$. The horizontal dashed lines show estimates of single-tail significance levels. The final session is shown for all subjects. 

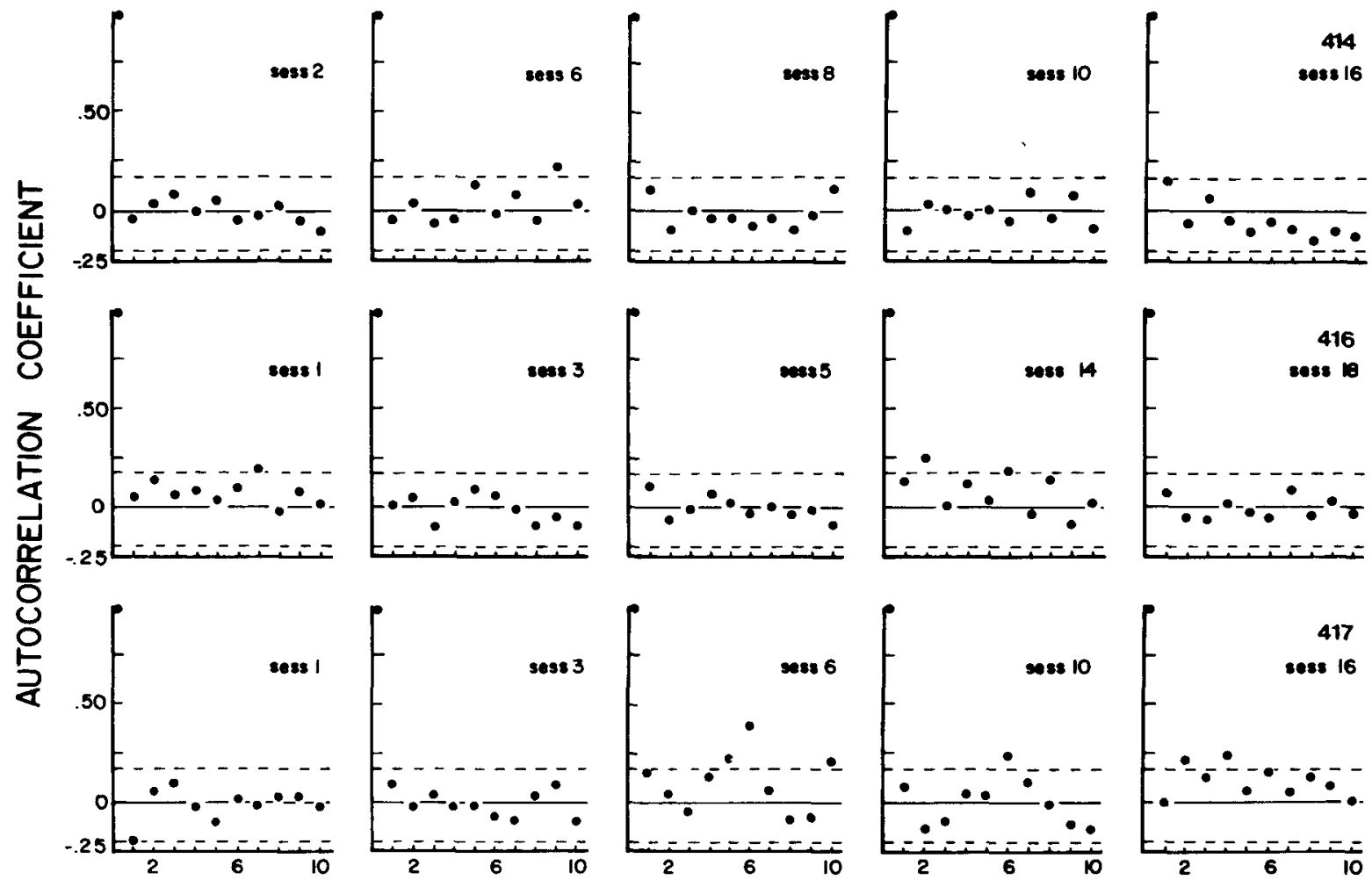

LAG

Figure 9. Autocorrelations up to a lag of 10 for selected time series in the green component during acquisition of concurrent VI $60 \mathrm{sec}$ (red) VI $120 \mathrm{sec}$ (green) for all subjects. Rows show data for individual subjects. Details are as in Figure 8 .

a statistically significant autocorrelation coefficient, shows no such obvious relationship. The distribution of data points at lag 6 appears to be somewhat less variable than it is at lag 1 . It is likely, then, that the significant correlation at lag 6 was due to the intrusion of a high correlation at lag 5 .

\section{DISCUSSION}

The present study analyzed intact time series generated during acquisition on several concurrent VI VI schedules. In several respects, overall terminal performances in this study replicated previous reports of molecular-level $\mathrm{CO}$ behavior. That is: (1) COs occurred with high frequency at certain intervals relative to the previous $\mathrm{CO}$ in the unpreferred of an asymmetrical pair of schedules (see Figures 1 and 2, cf. Hinson \& Staddon, 1981, 1983; Menlove, 1975; Silberberg et al., 1978); (2) overall probability of a $\mathrm{CO}$ response as a function of ICT was roughly constant (see Figure 5), as has been reported for both ICTs and run lengths (cf. Heyman, 1979a; Hinson \& Staddon, 1983; Silberberg et al., 1978); and (3) reinforcements occurred with high frequency immediately after the COD (cf. Menlove, 1975). In addition, the data in Figure 4 suggest that this temporal pattern of reinforcement is almost invariant across changes in schedule density and time allocation that occur during acquisition. The acquisition data also suggest that reductions in the variability of ICTs may be a typical response to a schedule-density manipulation. This result may have implications with regard to the behavioral mechanisms through which subjects make contact with changes in schedule contingencies and through which events are integrated over time.

While probability of a $\mathrm{CO}$ as a function of ICT was roughly constant, probability of a $\mathrm{CO}$ was an increasing function of time since reinforcement, in most cases. This is contrary to the report by Hinson and Staddon (1983), which suggests that pigeons do not "track" food deliveries. The reason for the difference between the two studies is not immediately obvious. It is reasonable to assume that reinforcement in a component could serve as a discriminative stimulus for nonavailability of reinforcement in that component for an interval equal to or greater than any minimum interreinforcement interval. Furthermore, reinforcement is infrequent and might be expected to be highly salient.

The lag transformations, both the scatter plots and the autocorrelograms, indicate that the relationship between adjacent ICTs is not preserved between 

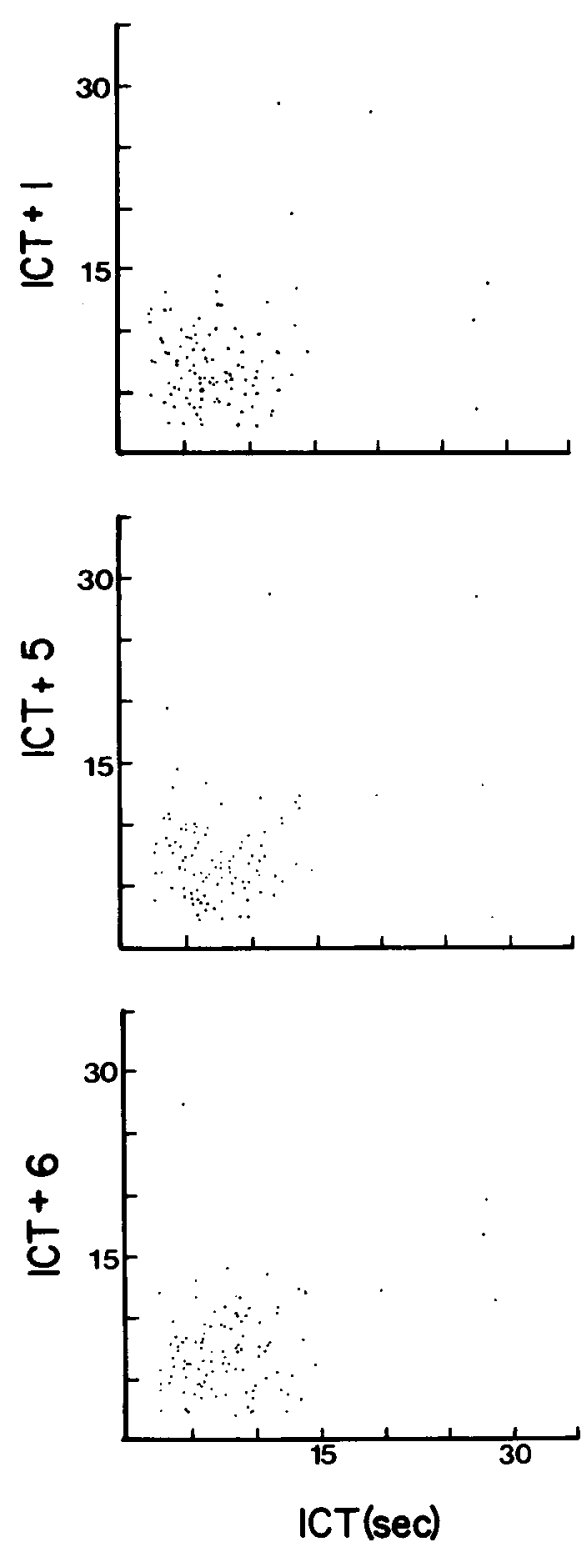

Figure 10. The duration of ICT $+n$ (in seconds) plotted as a function of ICT (in seconds) for Session 6 of concurrent VI $60 \mathrm{sec}$ (red) VI 120 sec (green) for Bird 417.

ICTs more remote from one another in the time series. This result suggests a type of nonstationarity to the time series that is not revealed by analysis of overall conditional probability of a CO (Heyman, 1979a). While the scatter plots shed some light on several types of temporal organization of ICTs that might have produced the significant autocorrelations, the reason for this type of organization in the time series is not transparent. That ICTs of similar duration should follow, or fail to follow, one another at different lags is not an entirely expected result. In general, however, the autocorrelograms confirm the fit of a Markov model (Heyman, 1979a) to the relationships among adjacent ICTs in the intact time series.

It is clear that molecular analyses of concurrent performance can provide consistent information about the local structure of $\mathrm{CO}$ responding and the local pattern of reinforcement that is not recoverable from more frequently used molar analyses. Perhaps the single most robust molar result to emerge from the study of concurrent schedules is that animals match (Herrnstein, 1970), that is, allocate time and responses to available alternatives in approximate proportion to reinforcements earned. Matching provides a quantitatively simple statement about a relation between performance and reinforcement. The theoretical importance of this relation has been the subject of much discussion (see Herrnstein, 1970; Nevin, 1979; Shimp, 1982), as has the relative merit of molar vs. molecular analysis (Fetterman \& Stubbs, 1982). It may be the case that molecular analyses afford no equivalently concise description of concurrent schedule behavior. However, in view of the complexities in molecular performance revealed by the present study and those that have preceded it (Hinson \& Staddon, 1981, 1983; Menlove, 1975; Silberberg et al., 1978), it appears clear that, at the very least, matching and other molar descriptions of concurrent performance do not reflect the subtleties of the behavioral stream that produce them.

\section{REFERENCES}

Anderson, R. L. Distribution of the serial correlation coefficient. Annals of Mathematical Statistics, 1942, 13, 1-13.

Blackman, R. B., \& Tukey, J. W. The measurement of power spectra. New York: Dover Press, 1958.

Dreyfus, L. R., Dorman, L. G., Fetterman, J. G., \& Stubis, D. A. An invariant relation between changing over and reinforcement. Journal of the Experimental Analysis of Behavior, $1982,38,327-338$.

Fetterman, J. G., \& Stubbs, D. A. Matching, maximizing, and the behavioral unit: Concurrent reinforcement of response sequences. Journal of the Experimental Analysis of Behavior, $1982,37,97-114$.

Fleshlen, M., \& Hoffman, H. S. A progression for generating variable-interval schedules. Journal of the Experimental Analysis of Behavior, 1962, 5, 529-530.

Herrnstein, R. J. On the law of effect. Journal of the Experimental Analysis of Behavior, 1970, 13, 243-266.

Herrnstein, R. J., \& Loveland, D. H. Maximizing and matching on concurrent ratio schedules. Journal of the Experimental analysis of Behavior, 1975, 24, 107-116.

HEYMAN, G. M. Markov model description of changeover probabilities on concurrent variable-interval schedules. Journal of the Experimental Analysis of Behavior, 1979, 31, 41-51. (a)

HEYMAN, G. M. Matching and maximizing in concurrent schedules. Psychological Review, 1979, 86, 496-500. (b)

Heyman, G. M., \& LuCE, R. D. Operant matching is not a logical consequence of maximizing reinforcement. Animal Learning \& Behavior, 1979, 7, 133-140.

Hinson, J. M., \& Staddon, J. E. R. Maximizing on interval schedules. In C. M. Bradshaw (Ed.), Recent developments in the 
quantification of steady-state operant behavior. Amsterdam: Elsevier/North-Holland, 1981.

Hinson, J. M., \& Staddon, J. E. R. Hill-climbing by pigeons. Journal of the Experimental Analysis of Behavior, 1983, 39, $25-47$.

Menlove, R. L. Local patterns of responding maintained by concurrent and multiple schedules. Journal of the Experimental Analysis of Behavior, 1975, 23, 309-337.

Nevin, J. A. Overall matching versus momentary maximizing: Nevin (1969) revisited. Journal of Experimental Psychology: Animal Behavior Processes, 1979, 5, 300-306.

Shimp, C. P. Optimal behavior in free-operant experiments. Psychological Review, 1969, 76, 97-112.

Shimp, C. P. Perspectives on the behavioral unit. In W. K. Estes (Ed.), Handbook of learning and cognitive processes (Vol. 2). Hillsdale, N.J: Erlbaum, 1975.
Sнiмp, C. P. Reinforcement and the local organization of behavior. In R. J. Herrnstein \& M. Commons (Eds.), Quantitative analyses of operant behavior (Vol. 2): Matching and maximizing accounts. New York: Ballinger, 1982.

Silberberg, A., Hamilton, B., Ziriax, J. M., \& Ca8ey, J. The structure of choice. Journal of Experimental Psychology: Animal Behavior Processes, 1978, 4, 368-398.

TintNe R, G. Econometrics. New York: Wiley, 1952.

Weiss, B., Laties, V. G., Siegal, L., \& Goldstein, D. A computer analysis of serial interactions in spaced responding. Journal of the Experimental Analysis of Behavior, 1966, 9, 619-626.

(Manuscript received August 12, 1982;

revision accepted for publication April 5, 1983.) 